\title{
Tracking of time-evolving sound speed profiles in shallow water using an ensemble Kalman-particle filter
}

\author{
Jianlong $\mathrm{Li}^{\mathrm{a})}$ and Hui Zhou \\ Department of Information Science and Electronic Engineering, Zhejiang University, \\ Hangzhou, 310027, China
}

(Received 18 October 2012; revised 19 December 2012; accepted 22 January 2013)

\begin{abstract}
This paper presents a tracking technique for performing sequential geoacoustic inversion monitoring range-independent environmental parameters in shallow water. The inverse problem is formulated in a state-space model with a state equation for the time-evolving sound speed profile (SSP) and a measurement equation that incorporates acoustic measurements via a hydrophone array. The particle filter $(\mathrm{PF})$ is an ideal algorithm to perform tracking of environmental parameters for nonlinear systems with non-Gaussian probability densities. However, it has the problem of the mismatch between the proposal distribution and the a posterior probability distribution (PPD). The ensemble Kalman filter (EnKF) can obtain the PPD based on the Bayes theorem. A tracking algorithm improves the performance of the PF by employing the PPD of the EnKF as the proposal distribution of the PF. Tracking capabilities of this filter, the EnKF and the PF are compared with synthetic acoustic pressure data and experimental SSP data. Simulation results show the proposed method enables the continuous tracking of the range-independent SSP and outperforms the PF and the EnKF. Moreover, the complexity analysis is performed, and the computational complexity of the proposed method is greatly increased because of the combination of the PF and the EnKF.
\end{abstract}

(C) 2013 Acoustical Society of America. [http://dx.doi.org/10.1121/1.4790354]

PACS number(s): 43.30.Pc, 43.60.Pt, 43.60.Wy, 43.60.Mn [ZHM]

Pages: $1377-1386$

\section{INTRODUCTION}

A typical ocean environment usually shows strong temporal variability, especially in shallow water. For example, many experiments conducted in shallow water have shown significant variability in the acoustic data caused by internal waves. ${ }^{1-5}$ Variability of the acoustic environment is one of the major obstacles to model based processing frameworks, such as matched field processing ${ }^{6-8}$ and matched field inversion. ${ }^{9}$

These variabilities usually span a wide range of spatial and temporal scales. It is not realistic for conventional oceanographic measurements to provide the ability to synoptically observe all these dynamic processes in shallow water, especially those with sub-mesoscale processes which are short in time and space.$^{10}$ Consequently, the coastal environment will always be under-sampled at these small and fast scales.

Ocean acoustic tomography (OAT) was suggested by Munk and Wunsch in the late 1970s, and is a powerful measurement tool to monitor large regions in the ocean environment. ${ }^{11}$ The sound speed profile (SSP) in the water column is an important source of information to extract ocean properties such as temperature or conductivity. It also is one of the most important parameters in determining acoustic waveguide propagation, for example, the existence of duct propagation or the fraction of energy interacting with the bottom. Numerous approaches have been developed to invert

\footnotetext{
${ }^{\text {a) }}$ Author to whom correspondence should be addressed. Also at: Woods Hole Oceanographic Institution, Woods Hole, MA 02543. Electronic mail: jlli@zju.edu.cn
}

for the sound speed in the water column by combining acoustic models with in situ measurements, such as the travel time approach in the early stages of the OAT, ${ }^{12}$ and matched field inversion ${ }^{9,13-17}$ in late years.

Oceanographic variabilities cause the SSP to evolve in time and space. The characteristics of these spatial or temporal variabilities can be reformulated as a tracking problem. The Kalman filter was first used to deal with a tracking problem under the assumption that the estimate is linear with Gaussian distributed noise. ${ }^{18}$ The extended Kalman filter (EKF) ${ }^{19}$ ensemble Kalman filter (EnKF) ${ }^{20,21}$ and particle filter $(\mathrm{PF})^{22,23}$ are subsequent approaches to handling nonlinear or (and) non-Gaussian estimation problems. Reference 17 is the first application of the EKF for inversion in ocean acoustics. Reference 24 tracks the sound speed field in a coastal water environment with empirical orthogonal functions (EOFs) describing the SSPs. Reference 25 is the first application of the EnKF to SSP inversion in a rangedependent environment. References 26 and 27 discussed the applications of the PF to track environment parameters with a moving source. An overview of sequential filtering in ocean acoustics was given in Ref. 28.

The Kalman filter (KF) is an exact method for a Gaussian distribution and a linear system. The EKF is an approximate filter for nonlinear systems based on first-order linearization. However, both the KF and the EKF need to maintain the state covariance matrix, which is not possible for large state dimensions. The EnKF replaces the covariance by the sample covariance computed from an ensemble of simulations. Hence, it can handle some problems where the dimensions of the states and observations are large. Since it uses a nonlinear model to propagate the ensemble states, it 
can be used to track nonlinear systems, but at the expense of incorrectly treating the non-Gaussian features of the forecast distribution that arise in nonlinear systems. Meanwhile, the EnKF is a Monte Carlo implementation of the Bayesian update problem, and can achieve the a posterior probability distribution (PPD) of the state of the modeled nonlinear system. On the other hand, the PF can represent non-Gaussian distributions and nonlinear systems faithfully, but it only updates the weights and cannot move ensemble members in the state space. This leads to the mismatch between the proposal distribution and the PPD of the nonlinear system and results in the well-known problem of sample degeneracy. Thus, as stated above, most filters have their own strengths and weaknesses. An approach that combines their advantages without their disadvantages is of interest.

Some efforts have been made to combine different types of filters. ${ }^{29-31}$ Reference 29 employed the EnKF to improve the weights of the PF. Reference 30 performed the EnKF and the PF in parallel and used the PF to improve the weights of the EnKF, with application to flood forecasting. In Ref. 31, the EKF was suggested to produce the proposal distribution of the PF in order to improve the performance of the PF. In terms of the PPD provided by the EnKF, this paper introduces a tracking algorithm for the ocean environment, which improves the performance of the PF by employing the PPD of the EnKF as the proposal distribution of the PF, and is named as the ensemble Kalman-particle filter (EnKPF) for simplicity.

It is known that the interaction between SSPs and complex acoustic pressures can involve a high level of nonlinearity, which leads to non-Gaussian PPDs of the tracking parameters. ${ }^{15,23}$ To properly resolve the profile recovery problem with nonlinear systems and non-Gaussian distributions, we use the EnKPF to track the time-evolving SSPs by simulating acoustic data measured by a vertical array. As a first step of the application of this new method to the SSP tracking, the ocean environment is assumed as rangeindependent. To reduce the degrees of freedom, EOFs are employed to describe the SSP perturbations. In the water column, EOFs are derived from direct measurements of the SSP and they are orthogonal in regard to the statistics of the SSP variations. $^{32}$ Tracking capabilities of the PF, the EnKF, and the EnKPF under slowly and quickly changing SSPs are compared in terms of divergence statistics with synthetic acoustic pressure data and experimental SSP data collected during the PRIMER experiment and the ASIAEX (Asian Seas International Acoustics Experiment) ECS (East China Sea) 2001 experiment, respectively. Because the running time is an important fact in the tracking problem, the complexity analysis of the algorithms is also performed.

\section{STATE-SPACE MODEL FOR SSP TRACKING}

As stated in Sec. I, the SSP in the water column can have significant effects on acoustic propagation in shallow water. It is, however, difficult to treat this as deterministic due to both its temporal and spatial variations. In the following, with the assumption of the range-independent environment, we describe random SSPs with EOFs, and take the
EOF coefficients as the state vector. Then, an equation modeling the evolution of EOF coefficients governed by the physical processes in shallow water is introduced. Moreover, the SSP tracking requires a measurement equation relating the observed acoustic field to the SSPs through a forward model, which is also presented in Sec. II B. These two dynamic equations characterize the system for SSP tracking.

\section{A. Empirical orthogonal function representation of SSP}

Tracking the SSP is a multi-dimensional optimization problem. The computational complexity and estimation accuracy of inversion algorithms usually depends on the number of estimated variables. To reduce the degrees of freedom, EOFs are employed to describe SSPs. EOFs are eigenvectors of the SSP covariance matrix which is usually estimated from onsite and/or historical SSP measurements. ${ }^{32}$ Each eigenvector represents one mode of the SSP variation with depth, while the corresponding eigenvalue indicates the amount of energy in that mode. A range-averaged SSP can be described by a mean profile, $\bar{c}(z)$ ( $z$ is depth), plus some zero-mean random perturbations, which are often expressed in terms of a set of EOFs, so that

$$
c(z)=\bar{c}(z)+\sum_{l=1}^{L} x_{l} \delta c_{l}(z)
$$

where $x_{l}$ is the $l$ th coefficient of EOFs satisfying

$$
x_{l}=\int_{0}^{D}[c(z)-\bar{c}(z)]^{T} \delta c_{l}(z) d z,
$$

and $\delta c_{l}(z)$ is the $l$ th EOF satisfying

$$
\int_{0}^{D} \delta c_{l_{1}}(z) \delta c_{l_{2}}(z) d z= \begin{cases}1 & l_{1}=l_{2} \\ 0 & l_{1} \neq l_{2}\end{cases}
$$

where $D$ is the water depth.

In practice, a limited number of EOFs are used to represent the SSP. The number and complexity of the EOFs that should be used depends on how detailed a profile is to be described, and what types of perturbations are considered possible. Ideally one should use the smallest number of parameters that are capable of capturing those important perturbation features. Generally, due to the red spectrum of the oceanography, the eigenvalues of the SSP covariance matrix diminish rapidly, so that only a few EOFs dominate the SSP characterization. Thus, the size of the EOF representation can be significantly reduced.

Figure 1(a) shows 26 samples of the SSPs measured during the shelf break PRIMER experiment, ${ }^{33,34}$ which was conducted on the shelf break south of New England in the Middle Atlantic Bight during summer 1996. The sound speeds were obtained from the onsite measurements along the range-independent track. ${ }^{34}$ They were sampled at 45 discrete depth points from the surface $(0 \mathrm{~m})$ to the bottom ( $88 \mathrm{~m}$ ), equally spaced by $2 \mathrm{~m}$. This spacing is chosen from the available SSP data measurements, and can certainly be 

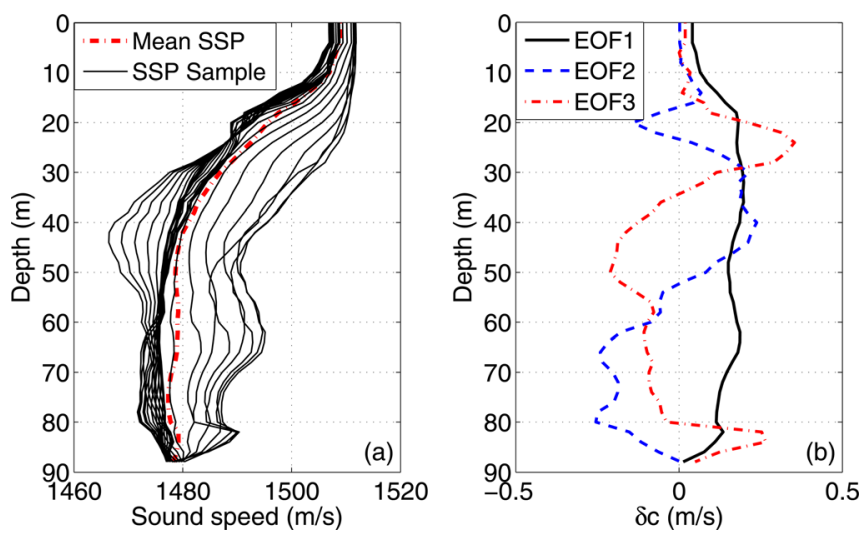

FIG. 1. (Color online) Sound speed profiles and the first three EOFs. (a) The SSPs measured in the shelf break PRIMER experiment (Refs. 33 and 34) and their average profile, (b) the first three EOFs.

larger for the purpose of representing either the SSP variations or the modal shape functions. The figure clearly shows that the SSPs fluctuate during the experiment. The greatest fluctuation amplitude compared with the mean SSP is $17.3 \mathrm{~m} / \mathrm{s}$ at $28 \mathrm{~m}$ in depth. The variability in water column sound speed is modeled using the EOFs shown in Fig. 1(b), where the first three EOFs are plotted. In terms of energy, these three describe $98.8 \%$ of the sound speed variation.

\section{B. The state equation and measurement equation}

It is known that the variation of SSPs is induced by the commonly dominant oceanographic processes. We assume that in a given region the rate of change of the associated EOF coefficients is slow, so that the coefficient evolution can be represented as a random walk. Considering $L$ EOFs, we define the state vector $\mathbf{x}_{k}=\left[\begin{array}{llll}x_{1 k} & x_{2 k} & \cdots & x_{L k}\end{array}\right]$. The state equation for step $k-1$ to step $k$ is given by

$$
\mathbf{x}_{k}=\mathbf{x}_{k-1}+\mathbf{v}_{k-1}
$$

where $k$ denotes the $k$ th time frame and $\mathbf{v}_{k-1}$ is the state noise vector for the EOF coefficients that takes into account the error in the evolution model, i.e., rapid changes in the SSP from step $k-1$ to step $k$. If the change in the state parameters is significantly different from the values given by the state equation, the filters may diverge, which usually occurs in the tracking of geoacoustic parameters at the boundaries of bathymetry features. ${ }^{24}$ The acoustic measurement equation relates the state vector to the observed acoustic pressure field. The field is usually measured across a receiver array. In this paper, the state vector is the EOF coefficients and the measurement vector is the complex acoustic pressures measured by a vertical linear array (VLA). The measurement equation can be formulated as

$$
\mathbf{y}_{k}=h\left(\mathbf{x}_{k}\right)+\xi_{k}
$$

where $\mathbf{y}_{k}$ is the complex acoustic pressure measured at the $k$ th time frame, $h(\cdot)$ is the acoustic propagation model, and $\xi_{k}$ is the measurement noise vector. For a single frequency signal, the dimension of the measurement vector $\mathbf{y}_{k}$ and noise vector $\xi_{k}$ is the same as the number of the hydro- phones. For a broadband signal with $\Omega$ discrete frequency bins, the dimension of $\mathbf{y}_{k}$ is $\Omega$ times the number of the hydrophones. This form of the expression is used to perform the coherent processing of the filters for a broadband signal. $\mathbf{v}_{k}$ and $\xi_{k}$ are assumed uncorrelated. The acoustic field across the VLA in the range-independent environment for an average SSP is calculated here by the normal mode equation model based on adiabatic normal modes. ${ }^{36}$ If the environment is range-dependent, a coupled mode model or a parabolic equation model should be used instead.

\section{ENSEMBLE KALMAN-PARTICLE FILTER AS A TRACKING PROCESSOR}

The state-space model formulation allows a straightforward implementation of sequential filters. In SSP tracking, the high nonlinearity of the measurement function $h(\cdot)$ requires nonlinear filtering methods. In Eqs. (4) and (5), both additive noise terms $\mathbf{v}_{k}$ and $\xi_{k}$ can adequately be represented by Gaussian probability density functions. However, the PPDs of the EOF coefficients are usually non-Gaussian, as shown in Refs. 15 and 24.

As discussed in Sec. I, the PF can perform nonlinear and non-Gaussian tracking, but it has the problem of sample degeneracy induced by the mismatch between the proposal distribution and the PPD. The EnKF is a Monte Carlo implementation of the Bayesian update problem and can obtain the PPD of the state of the modeled nonlinear system. In the following, we introduce the EnKPF which employs the PPD of the EnKF as the proposal distribution of the PF to improve the performance of the PF. Table I summarizes the principles of the EnKPF with the proposed state-space model, as described in Sec. II B. In a first step, the new state estimate $\mathbf{x}_{k, j}^{(i), b}$, correlation matrices $\hat{\mathbf{P}}_{x h}^{(i), k}$ and $\hat{\mathbf{P}}_{h h}^{(i), k}$ are calculated by Eqs. (4) and (5). In the second phase, each particle employs the EnKF to calculate the Kalman gain, $\mathbf{K}_{k}^{(i)}$, and the innovation, $\mathbf{E}_{k, j}^{(i)}$, in order to obtain the analysis ensemble (correction term), $\mathbf{X}_{k}^{(i), a}$, and its associated covariance matrix, $\hat{\mathbf{P}}_{k}^{(i), a}$. Then, one updates the particles, $\hat{\mathbf{x}}_{k}^{(i)}$, and weights, $w_{k}^{(i)}$, with the PPD produced by the mean value, $\overline{\mathbf{x}}_{k}^{(i), a}$, and the covariance matrix, $\hat{\mathbf{P}}_{k}^{(i), a}$, of the analysis ensemble, $\mathbf{X}_{k}^{(i), a}$. Finally, one estimates the state, $\hat{\mathbf{x}}_{k}$.

From the algorithm described in Table I, we find that the EnKPF is implemented in a PF framework, with the EnKF embedded into the PF to take the estimated PPD as the proposal distribution of the PF. This is shown in Fig. 2, where a diagram of tracking the time-evolving EOF coefficients by the EnKPF is depicted. The difference between the EnKPF and the standard PF is that each particle uses the EnKF independently to generate a new particle in the EnKPF.

\section{EXAMPLES}

This section is composed of two SSP tracking examples, one with slowly changing SSPs and one with fast changing SSPs. In the first example, we synthesize slowly changing SSPs with the EOFs shown in Fig. 1, and the associated pressure data with the geoacoustic parameters the same as reported for the acoustic environment of the PRIMER experiment. ${ }^{33,34}$

The second example employs the true SSP data and the geoacoustic parameters measured in the ASIAEX during 
TABLE I. Principles of the EnKPF applied to the state-space model.

Definitions

\begin{tabular}{|c|c|}
\hline $\mathbf{X}_{k}^{(i), b}:=\left\{\mathbf{x}_{k, j}^{(i), b}, j=1,2, \ldots, N_{e}\right\}$ & $\begin{array}{l}\text { Background ensemble member of the } \\
i \text { th particle, where } j \text { is the ensemble } \\
\text { member index and } k \text { is the time frame }\end{array}$ \\
\hline$\overline{\mathbf{x}}_{k}^{(i), b}$ & $\begin{array}{l}\text { Background ensemble mean of } \\
i \text { th particle }\end{array}$ \\
\hline $\mathbf{X}_{k}^{(i), a}:=\left\{\mathbf{x}_{k, j}^{(i), a}, j=1,2, \ldots, N_{e}\right\}$ & $\begin{array}{l}\text { Analysis ensemble member of } \\
i \text { th particle }\end{array}$ \\
\hline$\overline{\mathbf{x}}_{k}^{(i), a}$ & Analysis ensemble mean of $i$ th particle \\
\hline$\hat{\mathbf{x}}_{k}^{(i)}$ & The $i$ th particle \\
\hline$w_{k}^{(i)}$ & Weight of the $i$ th particle \\
\hline
\end{tabular}

$$
\begin{gathered}
\text { State prediction, mean value and covariance matrix } \\
\mathbf{x}_{k, j}^{(i), b}=\mathbf{x}_{k-1, j}^{(i), a}+\mathbf{v}_{k} \\
\overline{\mathbf{x}}_{k}^{(i), b}=\frac{1}{N_{e}} \sum_{j=1}^{N_{e}} \mathbf{x}_{k, j}^{(i), b} \\
\hat{\mathbf{P}}_{x h}^{(i), k}=\frac{1}{N_{e}-1} \sum_{j=1}^{N_{e}}\left(\mathbf{x}_{k, j}^{(i), b}-\overline{\mathbf{x}}_{k}^{(i), b}\right)\left(h\left(\mathbf{x}_{k, j}^{(i), b}\right)-h\left(\overline{\mathbf{x}}_{k}^{(i), b}\right)\right)^{T} \\
\hat{\mathbf{P}}_{h h}^{(i), k}=\frac{1}{N_{e}-1} \sum_{j=1}^{N_{e}}\left(h\left(\mathbf{x}_{k, j}^{(i), b}\right)-h\left(\overline{\mathbf{x}}_{k}^{(i), b}\right)\right)\left(h\left(\mathbf{x}_{k, j}^{(i), b}\right)-h\left(\overline{\mathbf{x}}_{k}^{(i), b}\right)\right)^{T} \\
\text { Ensemble of observations } \\
\mathbf{z}_{k, j}^{(i)}=\mathbf{y}_{k}+\varepsilon_{j}^{(i)}
\end{gathered}
$$

Ensemble covariance matrix

$$
\begin{gathered}
\mathbf{R}_{\varepsilon \varepsilon}^{(i), k}=\frac{1}{N_{e}-1} \sum_{j=1}^{N_{e}} \varepsilon_{j}^{(i)} \varepsilon_{j}^{(i) T} \\
\text { Kalman gain } \\
\mathbf{K}_{k}^{(i)}=\hat{\mathbf{P}}_{x h}^{(i), k}\left(\hat{\mathbf{P}}_{h h}^{(i), k}+\mathbf{R}_{\varepsilon \varepsilon}^{(i), k}\right)^{-1} \\
\text { Innovation } \\
\mathbf{E}_{k, j}^{(i)}=\mathbf{z}_{k, j}^{(i)}-h\left(\mathbf{x}_{k, j}^{(i), b}\right) \\
\text { Correction } \\
\mathbf{x}_{k, j}^{(i), a}=\mathbf{x}_{k, j}^{(i), b}+\mathbf{K}_{k}^{(i)} \mathbf{E}_{k, j}^{(i)} \\
\overline{\mathbf{x}}_{k}^{(i), a}=\frac{1}{N_{e}} \sum_{j=1}^{N_{e}} \mathbf{x}_{k, j}^{(i), a} \\
\hat{\mathbf{P}}_{k}^{(i), a}=\frac{1}{N_{e}-1} \sum_{j=1}^{N_{e}}\left(\mathbf{x}_{k, j}^{(i), a}-\overline{\mathbf{x}}_{k}^{(i), a}\right)\left(\mathbf{x}_{k, j}^{(i), a}-\overline{\mathbf{x}}_{k}^{(i), a}\right)^{T} \\
\text { Update particles and weights } \\
\hat{\mathbf{x}}_{k}^{(i)} \sim q\left(\hat{\mathbf{x}}_{k}^{(i)} \mid \mathbf{x}_{k-1}^{(i)}, \mathbf{y}_{k}\right)=N\left(\overline{\mathbf{x}}_{k}^{(i), a}, \hat{\mathbf{P}}_{k}^{(i), a}\right) \\
w_{k}^{(i)}=w_{k-1}^{(i)} \frac{p\left(\mathbf{y}_{k} \mid \hat{\mathbf{x}}_{k}^{(i)}\right) p\left(\hat{\mathbf{x}}_{k}^{(i)} \mid \mathbf{x}_{k-1}^{(i)}\right)}{q\left(\hat{\mathbf{x}}_{k}^{(i)} \mid \mathbf{x}_{0 i k-1}^{(i)}, \mathbf{y}_{1: k}\right)} \\
\text { State estimation } \\
\hat{\mathbf{x}}_{k}=\sum_{i=1}^{N_{p}} w_{k}^{(i)} \hat{\mathbf{x}}_{k}^{(i)}, \text { where } N_{p} \text { is the number of particles }
\end{gathered}
$$

June $2001 ;^{35}$ the associated pressure data are also synthesized. In this example, the SSPs in some time intervals changed quite fast, so it can be used to test the performance of the EnKPF in the case of quickly time-evolving SSPs. Both examples evaluate and compare different aspects of the PF, EnKF, and EnKPF.

\section{A. Example 1: Tracking of slowly changing SSPs}

\section{Simulation setup}

The simulation environment for example 1 is shown in Fig. 3 and the EOFs are presented in Fig. 1. The environment parameters in water column were measured from the PRIMER experiment and the sediment parameters were inverted from the observed broadband acoustic signals. ${ }^{33}$ The water depth is $88 \mathrm{~m}$. In the sediment, the sound speed, attenuation, and density are $c_{\text {bot }}=1750 \mathrm{~m} / \mathrm{s}, \alpha_{\mathrm{bot}}=0.05 \mathrm{~dB} / \lambda$, and $\rho_{\text {bot }}=1.7 \mathrm{~g} / \mathrm{cm}^{3}$, respectively. To synthesize the pressure data used in the measurement equation, a source and a VLA are added in this environment, as listed in Table II. The VLA consists of 16 elements deployed from $15 \mathrm{~m}$ to $75 \mathrm{~m}$ in depth, spaced $4 \mathrm{~m}$ apart. The source is placed at $30 \mathrm{~m}$ in depth and $5 \mathrm{~km}$ in range with a frequency of $400 \mathrm{~Hz}$. The covariance of the measurement error term $\mathbf{R}_{\xi_{\xi}}=\sigma^{2} \mathbf{I}$ is evaluated from the array signal-to-noise ratio (SNR), which is used to synthesize the observed acoustic data.

\section{Tracking of the SSPS}

To perform the comparison of the EnKPF with the PF and the EnKF, we take the particle number and the ensemble number as 20 and 5, respectively. These numbers are related to the precision of the associated filters, but the same values are used in the comparison. The tracking length is $12 \mathrm{~h}$ and the tracking interval is $5 \mathrm{~min}$. The tracking interval can be adjusted according to practical requirements. Full-wave synthetic pressure fields were generated using a normal mode code. $^{36}$ The algorithms are first initialized with random perturbations, which do not affect the stable performance when the filters converge. The evolution of the three EOF coefficients is seen in Fig. 4, where the tracking results of the SSPs at 2-h increments and their relative errors amplified by 100 times are also shown. The time-evolving SSPs are tracked using the PF, EnKF, and EnKPF, where the first two filters are used to give a comparison to the performance of the EnKPF. It is seen from the figure that all three filters are sensitive to the EOF coefficients and are able to track timeinvolving SSPs. Moreover, by comparison, the track given by the EnKPF is superior to the other two, which is especially obvious from the relative errors of the SSPs in the last row.

The errors in the SSP estimation can be evaluated by the depth-integrated root mean square error (RMSE) metric, which essentially calculates the difference between the true sound speed and the estimated sound speed from the tracker and then integrates across these values in depth to provide a single number defined by

$$
\Delta_{c_{\mathrm{RMS}}}=\sqrt{\frac{1}{D} \sum_{z=z_{1}}^{z=z_{D}}\left[c_{\text {true }}(z)-c_{\mathrm{est}}(z)\right]^{2}} .
$$

By examining the depth-integrated RMSE evolution, the divergence in the filters can be seen more obviously. As shown in Fig. 5, all the three filters converge quickly and $\Delta c_{\mathrm{RMS}}$ becomes stable in less than teniterations. From Fig. 5, it is confirmed that the EnKPF outperforms the PF and EnKF over the whole tracking period.

To quantify the average tracking performance, Table III gives the time-average and depth-integrated RMSE of the 


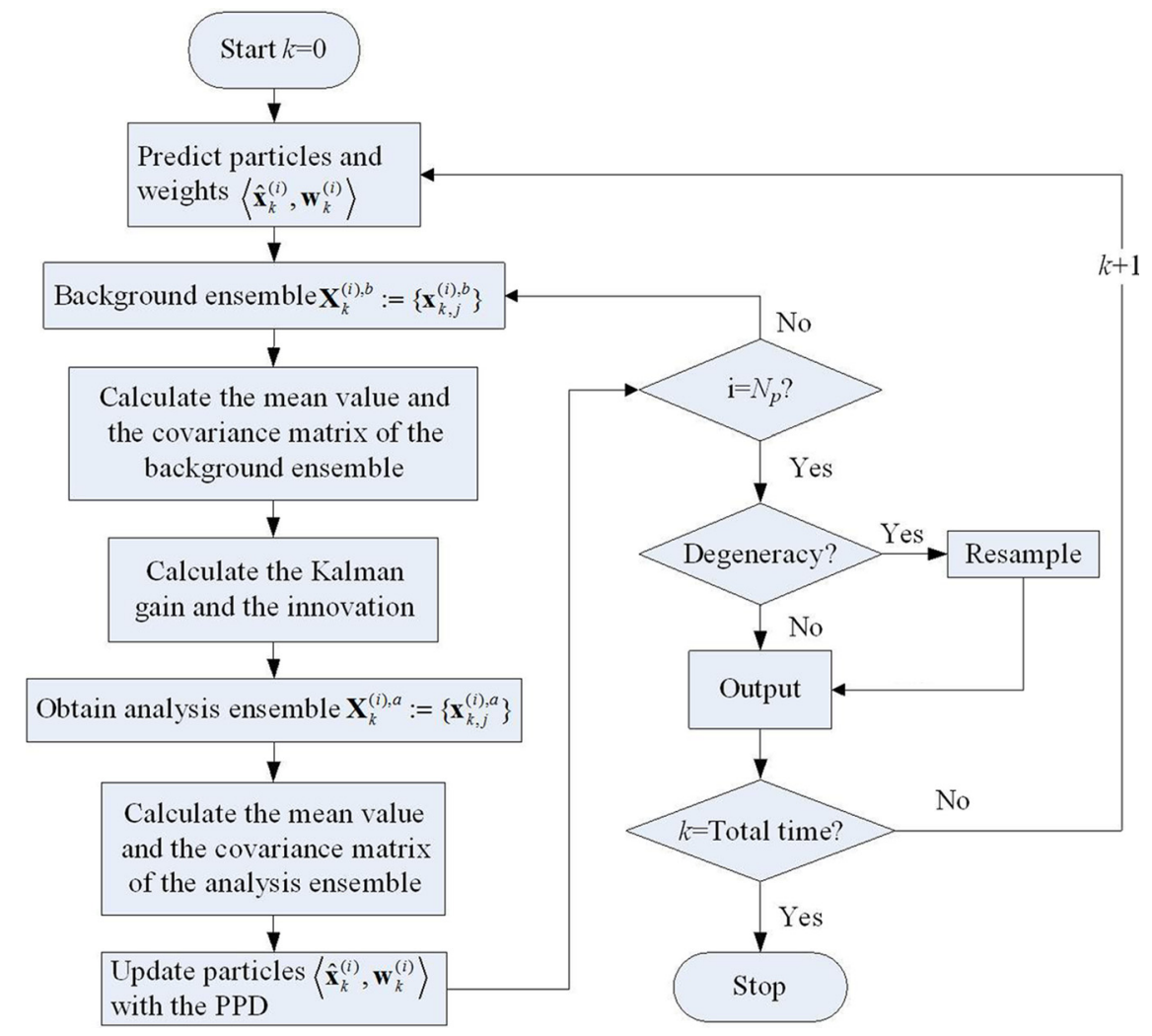

FIG. 2. (Color online) Diagram of tracking time-evolving EOF coefficients by the EnKPF.

three filters. We find that the value of the EnKPF is half the value of the EnKF and less than 1/3 the value of the PF.

\section{Impact of different SNRs}

To investigate the robustness of the EnKPF for different measured acoustic pressure noise levels, Fig. 6 gives the depth-integrated RMSE for different array SNRs. The timeaverage and depth-integrated RMSE is listed in Table IV. As expected, the RMSE increases with the decrease of the SNR. The EnKPF still successfully tracks the time evolving SSP and has an acceptable value $0.25 \mathrm{~m} / \mathrm{s}$ of the time-averaged and depth-integrated RMSE when the array SNR is $10 \mathrm{~dB}$.

\section{Effects of array configurations and signal frequencies}

The configuration used in the above simulation is a 16element VLA. It is of practical interest to investigate whether the tracking method is robust when using only a few

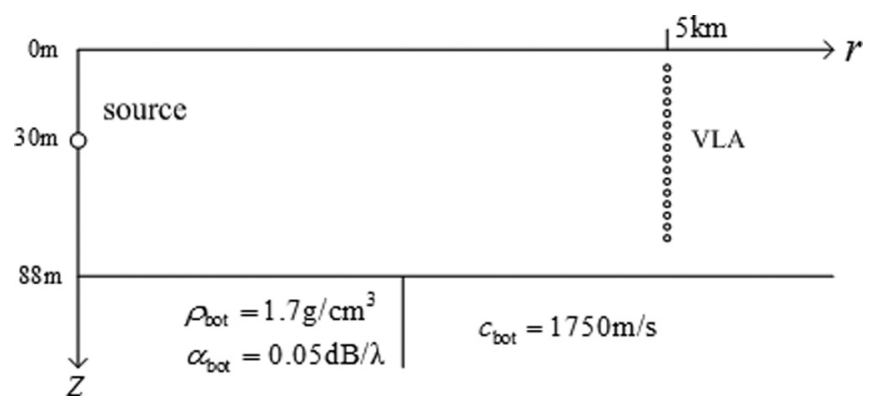

FIG. 3. The geoacoustic model used in the simulations for example 1, where the water depth and sediment parameters are the same as the PRIMER experiment (Refs. 33 and 34). hydrophones. The initial signal frequency $400 \mathrm{~Hz}$ is a single frequency. However, broadband signals are also widely used in geoacoustic inversion. As proposed in the model-based matched filter technique, ${ }^{37}$ an increase in the number of frequency bins can compensate for a lack of hydrophones on the VLA. The different configurations tested are given in Table V. The performances of the EnKPF are summarized through the evolution of the depth-integrated RMSE in Fig. 7 and the time-average and depth-integrated RMSE in Table VI. Unsurprisingly, Test B outperforms Test A and Test $\mathrm{C}$ because it has the maximum number of measurements $(16 \times 2)$ among the three tests, which shows that either the increase of the number of frequency bins or the increase of the number of hydrophones can improve the tracking accuracy of the EnKPF. Examining Test A and Test $\mathrm{C}$, it also appears that the performance of the filter in these two cases is quite comparable. Such results may be explained by noting that the number of given measurements in both two tests is 16. It should be pointed out that the equal number of measurements does not mean the same performance of the filter. The independence of the measurements is also a key factor that affects the performance.

TABLE II. Simulation parameters of the source and the received array for example 1 .

\begin{tabular}{ll}
\hline \hline Source frequency & $400 \mathrm{~Hz}$ \\
Source depth & $30 \mathrm{~m}$ \\
Source range & $5 \mathrm{~km}$ \\
Number of hydrophones & 16 \\
Array start, element spacing & $15 \mathrm{~m}, 4 \mathrm{~m}$ \\
Array SNR & $30 \mathrm{~dB}$ \\
\hline \hline
\end{tabular}


PF

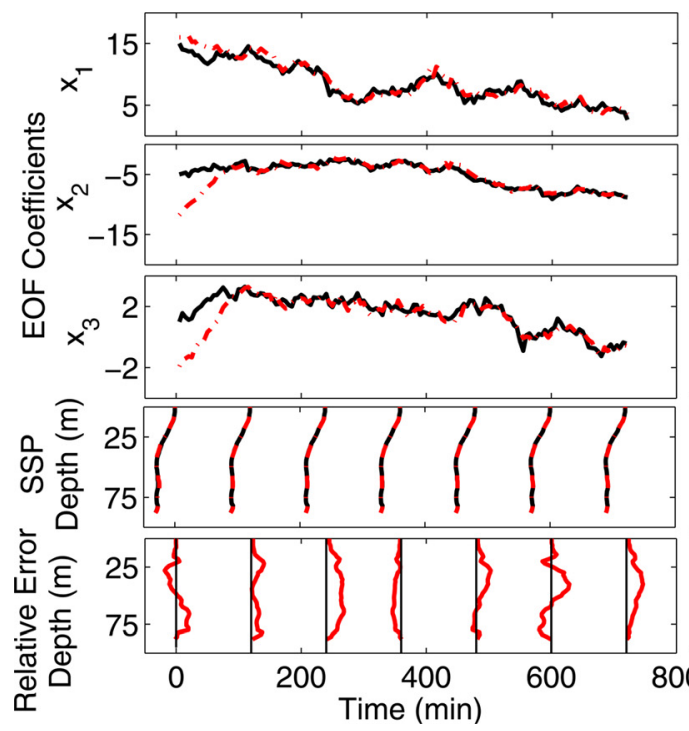

EnKF
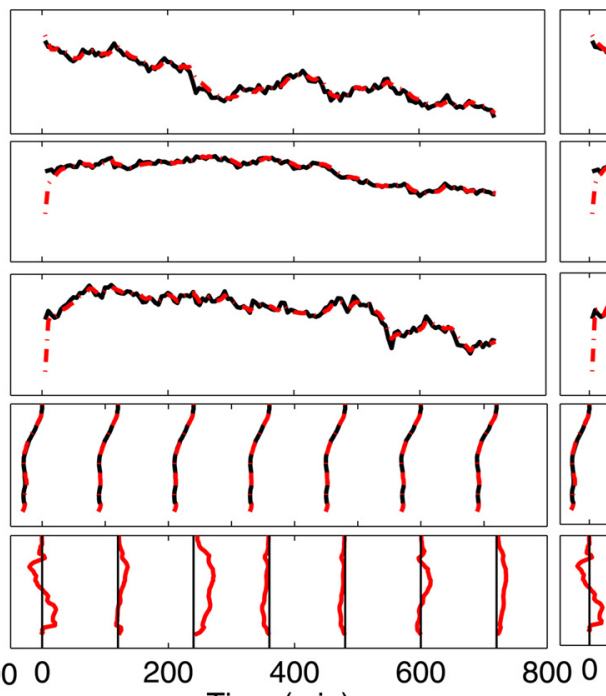

Time (min)

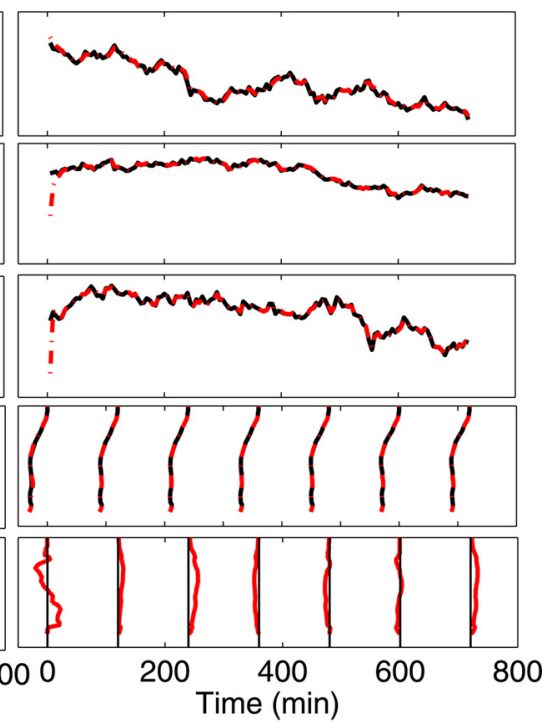

EnKPF

FIG. 4. (Color online) Example 1: Tracking results of the PF, EnKF, and EnKPF. The solid lines are the true trajectories and the dashed-dotted lines denote the tracking results of the filters. The first three rows show the tracking of the three EOF coefficients, the fourth row shows the tracking of SSPs at 2-h increments, and the last row shows the relative errors (amplified by 100 times) of the associated SSPs shown in the fourth row.

\section{B. Example 2: Tracking of fast changing SSPs}

To test the performance of the filters in the case of fast changing SSPs, the SSP data measured in the 2001 ASIAEX were used. The ASIAEX was a multinational scientific project conducted in both the East China Sea (ECS) and the South China Sea. ${ }^{35}$ The ECS part of the ASIAEX, which is the part on which we will focus here, was conducted from May 27 to June $9,2001 .^{38-40}$ One of the main goals of the ASIAEX ECS component was to contribute to a more fundamental understanding of ocean acoustic propagation and scattering (reverberation) in shallow water. It involved acoustic, oceanographic, and geological field measurements. Measurements of the SSP data used here were made in the ECS from 19:35, June 2, to 19:35, June 6, 2001 (GMT8 time zone). The experiment site was shown in Refs. 35 and 38-40. Ninety-six hours of data were recorded by conductivity, temperature, and depth (CTD) sensors casts at $12649.30^{\prime} \mathrm{E}, 2940.54^{\prime} \mathrm{N}$ (all the data were recorded in an area of less $2 \mathrm{~km}$ in diameter). In Fig. 8 , an ensemble of 53

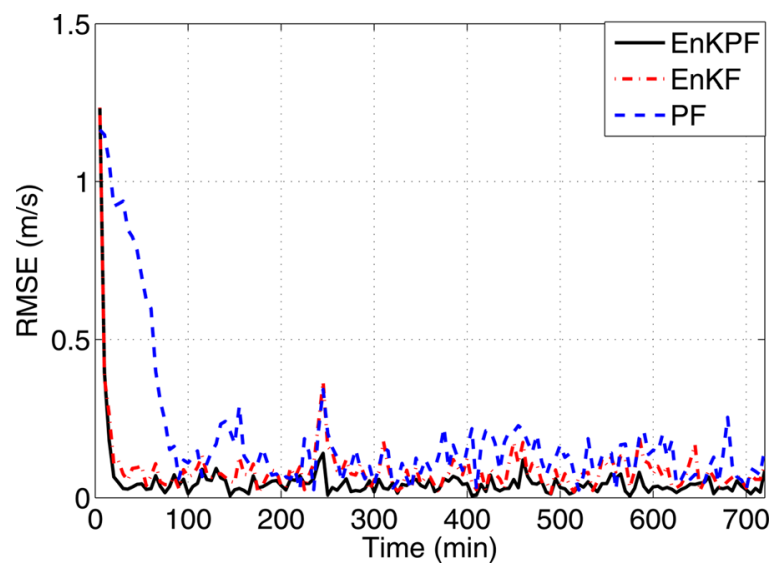

FIG. 5. (Color online) Depth-integrated RMSE of the EnKPF (solid line), the EnKF (dashed-dotted line), and the PF (dashed line) with $30 \mathrm{~dB}$.
CTD casts and their average values illustrate the typical variation in sound speed as a function of depth for the experimental area. Figure 8 also plots the first three EOFs, which, in terms of energy, describe $88.7 \%$ of the sound speed variation. A contour of this data (Fig. 9) shows how conditions changed over the course of the experiment. Variation linked with the fast changing waves (mixed) is evident during the period June 2-3, and high winds are likely responsible for more water column mixing during the period June 4-6. The data were sampled every $1-2 \mathrm{~h}$, and they are shown in Fig. 9 by a linear interpolation for an equal sampling interval. The interpolation calculation should not affect the performance demonstration of the filters.

To synthesize the acoustic pressure data used in the measurement equation, the following simulation configuration is used. The water depth is $106 \mathrm{~m}$ according to the measurement. The sediment was assumed to be a homogenously acoustic halfspace with a sound speed of $1610 \mathrm{~m} / \mathrm{s}$, density of $1.7 \mathrm{~g} / \mathrm{cm}^{3}$, and attenuation rate of $0.05 \mathrm{~dB} / \lambda$. These values correspond to average values deduced from the core measurements. ${ }^{41}$ The parameters of the VLA are the same as those listed in Table II. The source is placed at $30 \mathrm{~m}$ in depth and $5 \mathrm{~km}$ in range with two frequency bins of $400 \mathrm{~Hz}$ and $600 \mathrm{~Hz}$ and SNR of $30 \mathrm{~dB}$.

The evolution of the three EOF coefficients is presented in Fig. 10, where the results of SSP tracking every $12 \mathrm{~h}$ are also given. We find that all three filters can track the

TABLE III. Time-averaged and depth-integrated RMSE of different filters with $30 \mathrm{~dB}$.

\begin{tabular}{lc}
\hline \hline Filter & Time-averaged and depth-integrated RMSE $(\mathrm{m} / \mathrm{s})$ \\
\hline PF & 0.13 \\
EnKF & 0.08 \\
EnKPF & 0.04 \\
\hline \hline
\end{tabular}




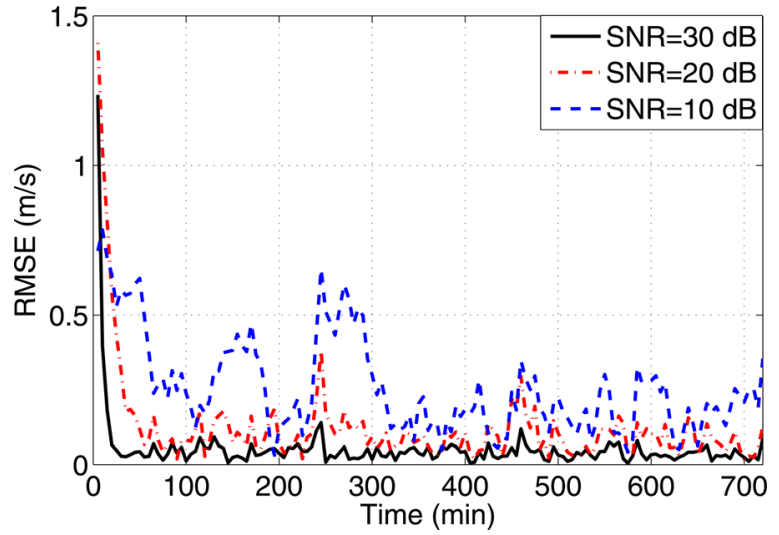

FIG. 6. (Color online) Depth-integrated RMSE of the EnKPF for different SNRs with $30 \mathrm{~dB}$ (solid line), $20 \mathrm{~dB}$ (dashed-dotted line), and $10 \mathrm{~dB}$ (dashed line).

TABLE IV. Time-averaged and depth-integrated RMSE of the EnKPF for different array SNRs.

\begin{tabular}{lc}
\hline \hline SNR $(\mathrm{dB})$ & Time-averaged and depth-integrated RMSE (m/s) \\
\hline 30 & 0.04 \\
20 & 0.10 \\
10 & 0.25 \\
\hline \hline
\end{tabular}

TABLE V. Simulated configurations with different hydrophones and frequencies.

\begin{tabular}{lcccc}
\hline \hline & $\begin{array}{c}\text { Number of } \\
\text { hydrophones }\end{array}$ & Depths & $\begin{array}{c}\text { Number of } \\
\text { frequencies }\end{array}$ & $\begin{array}{c}\text { Frequency } \\
(\mathrm{Hz})\end{array}$ \\
\hline Test A & 16 & $15 \mathrm{~m}-75 \mathrm{~m}, 4 \mathrm{~m}$ spacing & 1 & 400 \\
Test B & 16 & $15 \mathrm{~m}-75 \mathrm{~m}, 4 \mathrm{~m}$ spacing & 2 & 400,600 \\
Test C & 8 & $15 \mathrm{~m}-75 \mathrm{~m}, 7.5 \mathrm{~m}$ spacing & 2 & 400,600 \\
\hline \hline
\end{tabular}

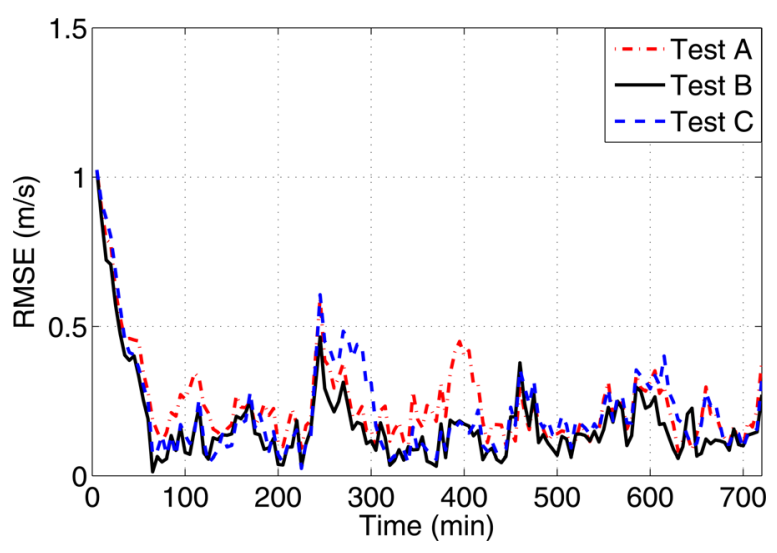

FIG. 7. (Color online) Depth-integrated RMSE of the EnKPF for different tests (Test A: dashed-dotted line, Test B: solid line, and Test C: dashed line).

TABLE VI. Time-average and depth-integrated RMSE of the EnKPF for different tests in Table VI.

\begin{tabular}{lc}
\hline \hline & Time-average and depth-integrated RMSE (m/s) \\
\hline Test A & 0.21 \\
Test B & 0.13 \\
Test C & 0.17 \\
\hline \hline
\end{tabular}
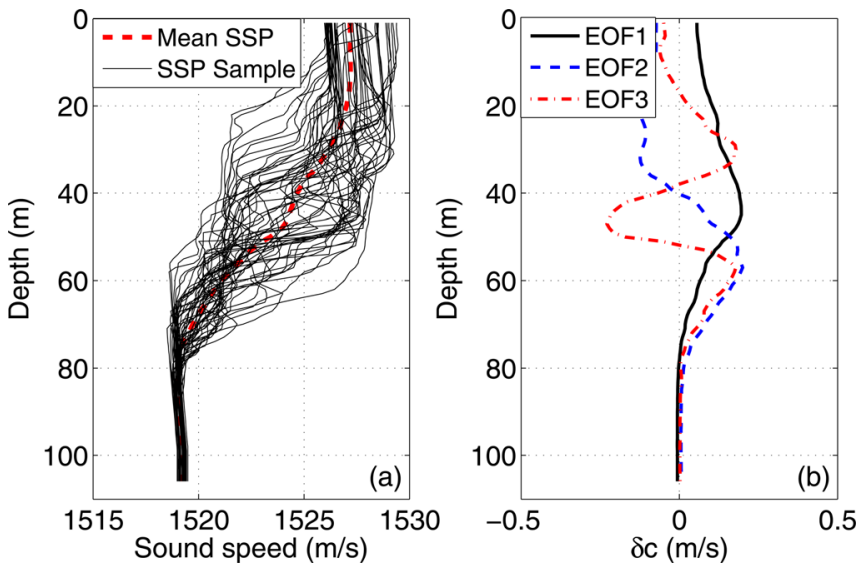

FIG. 8. (Color online) The sound speed profiles of 53 CTD casts and (a) their average profile and (b) the first three EOFs.

time-evolving EOF coefficients. The EnKPF again outperforms the PF and the EnKF, which is quite obvious from the relative errors shown in the last row. Moreover, from Fig. 10 we find that the errors are relatively large for most of the fast changing region of the EOF coefficients, especially for the $\mathrm{PF}$ and EnKF. It is seen from Fig. 10 that the tracking of the third EOF coefficient $x_{3}$ is worse than the other two, and is quite obvious in the tracking of the PF. The possible reason is that the third EOF plays a minor position compared with the first two on dominating the SSP characterization, which leads to a minor variation of the SSP in Eq. (1) and little pressure variation information ${ }^{7}$ (low associated SNR) in measured acoustic pressure data to help the filter to well catch the variation of $x_{3}$. Moreover, from the figure of the SSP tracking, the errors near the thermocline are large compared with other regions because SSPs change fast in this region.

Figure 11 shows the depth-integrated RMSE evolution. Although all three filters still converge, the depth-integrated RMSEs are much larger than those in example 1. The values near $20 \mathrm{~h}$ are especially large (close to $2 \mathrm{~m} / \mathrm{s}$ for the PF), which can be predicted by Fig. 10, where the EOF coefficients at that time change quickly. The figure again displays that the EnKPF outperforms the PF and EnKF over the whole tracking period. Table VII lists the time-averaged and

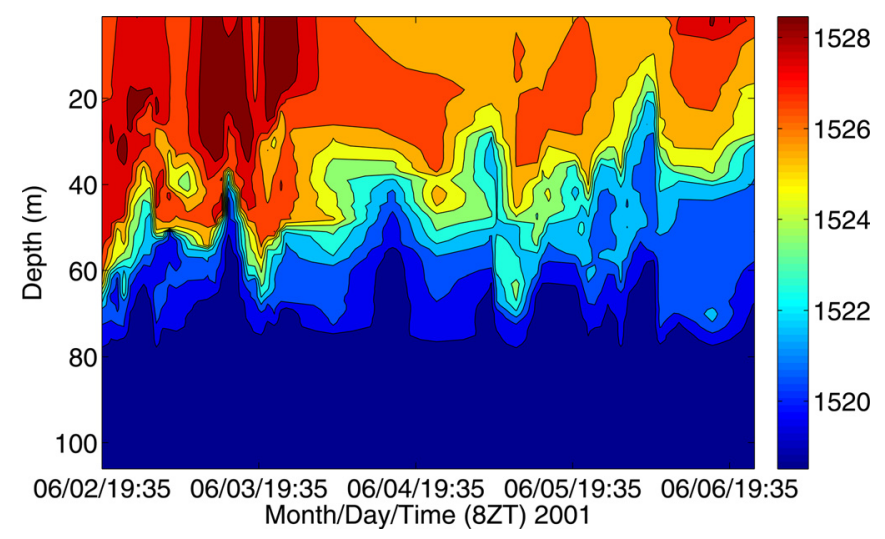

FIG. 9. (Color online) Time-evolving sound speed profiles calculated from 53 CTD casts made every $1--2 \mathrm{~h}$ during the ASIAEX ECS 2001 experiment. 
PF

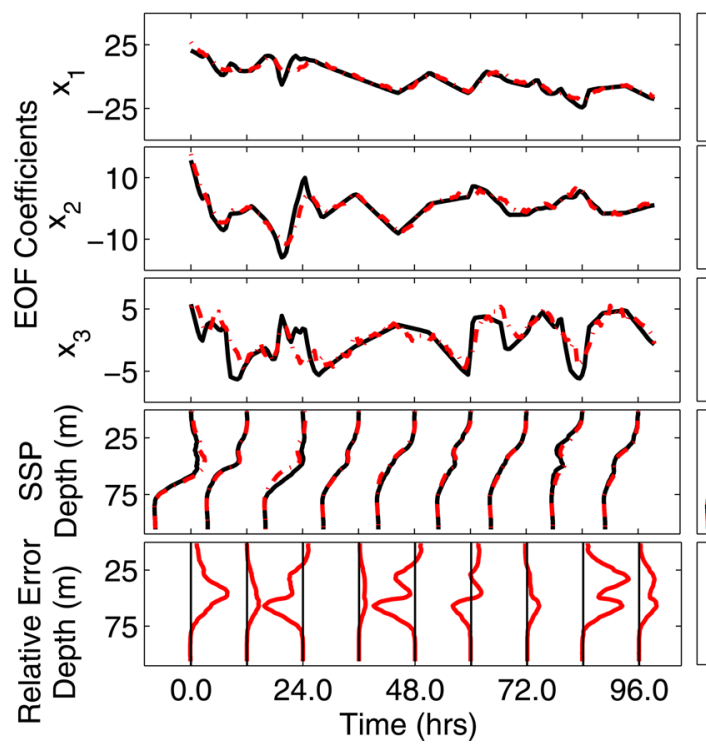

EnKF

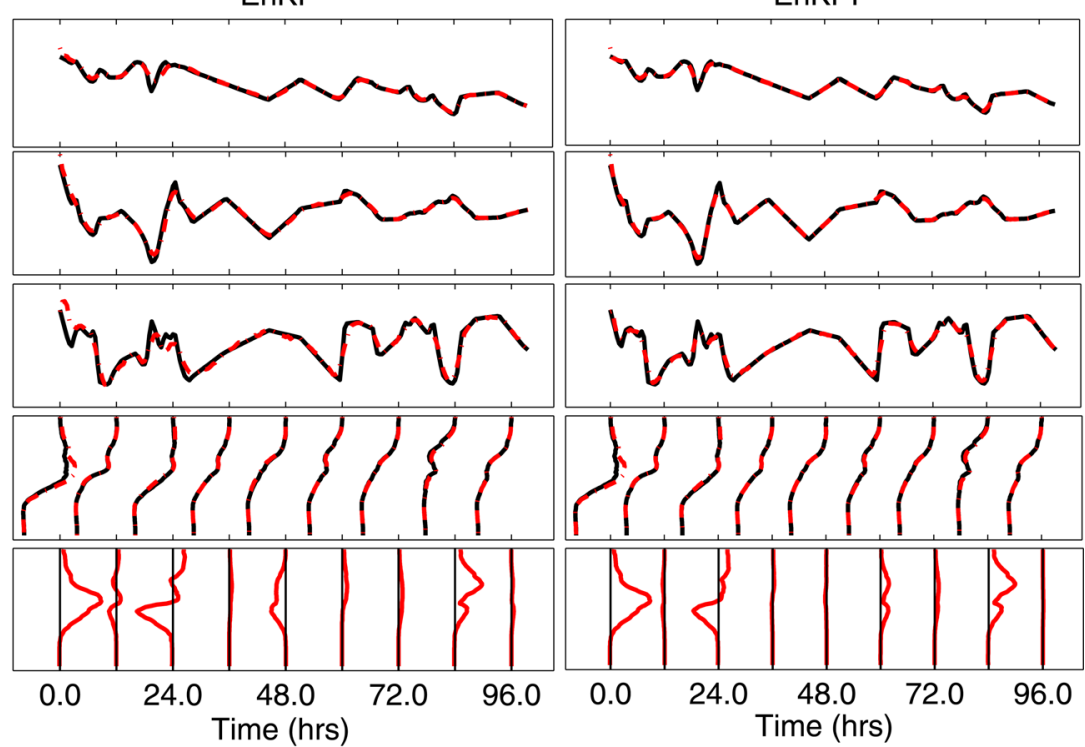

FIG. 10. (Color online) Example 2: Tracking results of the PF, EnKF, and EnKPF. The solid lines are the true trajectories and the dashed-dotted lines denote the tracking results of the filters. The first three rows are the tracking of the three EOF coefficients, the fourth row shows the tracking of the SSPs every $12 \mathrm{~h}$, and the last row shows the relative errors (amplified by 10 times) of the associated SSPs presented in the fourth row.

depth-integrated RMSE of the three filters, which confirms that the EnKPF is much better than the PF and EnKF.

\section{COMPLEXITY ANALYSIS}

Section IV shows that the EnKPF outperforms the PF and the EnKF from the viewpoint of the tracking of the time-evolving SSP. As a tracking algorithm, the computational cost is important too. Because the running time depends on the hardware of the computer, in this section we employ the computational complexity to evaluate the efficiency of the algorithms by giving the number of floatingpoint operations (flops) used in the algorithms. A flop is defined as one addition, subtraction, multiplication, or division of two floating-point numbers. Although there are some drawbacks, for example, some steps in the algorithms cannot easily be measured in flops, it is extensively used to analyze the complexity using the computer to measure the absolute time that the different steps require. ${ }^{42}$

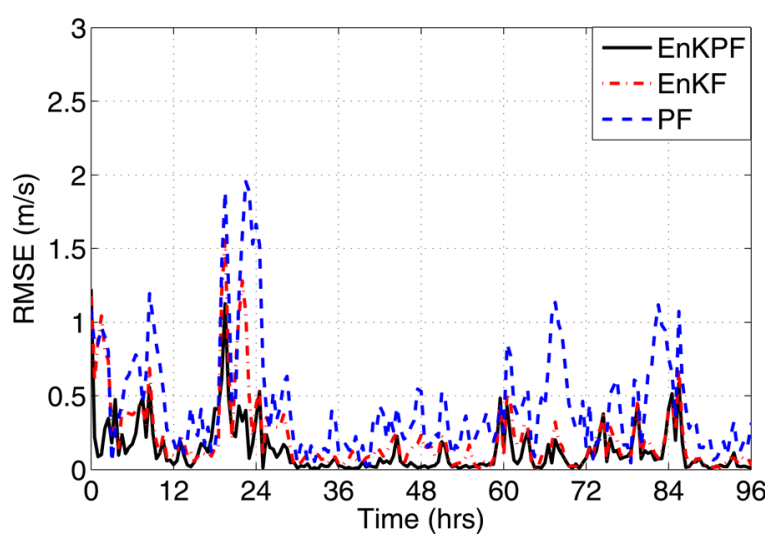

FIG. 11. (Color online) Depth-integrated RMSE of the PF (dashed line), the EnKF (dashed-dotted line), and the EnKPF (solid line) for example 2.
According to Sec. II, the number of the state estimation is $L$, and here we assume the size of the measurement vector is $M$. The addition of matrices of size $M$ by $M$ costs $O\left(M^{2}\right)$. The multiplication of matrices of size $L \times M$ and $M \times M$ costs $O\left(L M^{2}\right)$. The inverse of matrix of size $M \times M$ costs $O\left(M^{3}\right) .^{42}$ The straightforward implementation of the EnKPF listed in Table I leads to the computational complexity shown in Table VIII. From Table VIII, we can evaluate that the total computational complexity of the EnKPF is $O\left(N_{p}\left(N_{e}\left(L M+M^{2}+L^{2}\right)+\left(M^{3}+M^{2}+L M^{2}+L \mu\right)\right)\right)$, where $\mu$ is the random number complexity. For a normal desktop computer running MATLAB, $\mu=125$ [on a Sun Blade 100 with 640-MB memory (Oracle Corp., Redwood Shores, CA)]. ${ }^{42}$ In terms of the operations of the PF and the EnKF, it is easy to estimate that their computational complexities are $O\left(N_{p}(L \mu+M)\right)$ and $O\left(N_{e}\left(L M+M^{2}+L^{2}\right)+\left(M^{3}+M^{2}+L M^{2}\right)\right)$, respectively. For the two examples discussed in the Sec. IV, $N_{p}=20$, $N_{e}=5, L=3$, and $M=16$, we can calculate that the computational complexities of the PF and the EnKF are comparable $\left[N_{p}(L \mu+M)=7820\right.$ for the PF and $N_{e}\left(L M+M^{2}+L^{2}\right)$ $+\left(M^{3}+M^{2}+L M^{2}\right)=6685$ for the EnKF], and the computational complexity of the EnKPF is greatly increased, about 18 times as compared to that of the PF. Therefore, it should be pointed out that the EnKPF has the best accuracy in the tracking process, but its computational complexity is greatly increased because of the combination of the PF and the EnKF. In practice, based on the hardware employed to run the algorithms, the filter performance and the running time should be weighted.

TABLE VII. Time-averaged and depth-integrated RMSE of different filters for example 2 .

\begin{tabular}{lc}
\hline \hline Filter & Time-averaged and depth-integrated RMSE $(\mathrm{m} / \mathrm{s})$ \\
\hline $\mathrm{PF}$ & 0.31 \\
EnKF & 0.13 \\
EnKPF & 0.08 \\
\hline
\end{tabular}


TABLE VIII. Computational complexity of the EnKPF listed in Table I.

\begin{tabular}{|c|c|c|c|}
\hline Computation & Operation & Size & Cost \\
\hline State prediction $\mathbf{x}_{k, j}^{(i), b}$ & Vector add & $(L \times 1)+(L \times 1)$ & $O\left(N_{p} N_{e} L\right)$ \\
\hline Background ensemble mean $\overline{\mathbf{x}}_{k}^{(i), b}$ & Vector add & $(L \times 1)+(L \times 1)$ & $O\left(N_{p} N_{e} L\right)$ \\
\hline Measurement prediction $h\left(\overline{\mathbf{x}}_{k, j}^{(i), b}\right)$ & Vector add & $(M \times 1)+(M \times 1)$ & $O\left(N_{p} N_{e} M\right)$ \\
\hline Background covariance matrices $\hat{\mathbf{P}}_{x h}^{(i), k}$ & Vector multiply and add & $(L \times 1) \times(1 \times M)$ & $O\left(N_{p} N_{e} L M\right)$ \\
\hline Background covariance matrices $\hat{\mathbf{P}}_{h h}^{(i), k}$ & Vector multiply and add & $(M \times 1) \times(1 \times M)$ & $O\left(N_{p} N_{e} M^{2}\right)$ \\
\hline Measurement error covariance matrix $\mathbf{R}_{\varepsilon \varepsilon}^{(i), k}$ & Vector multiply and add & $(M \times 1) \times(1 \times M)$ & $O\left(N_{p} N_{e} M^{2}\right)$ \\
\hline \multirow[t]{3}{*}{ Kalman gain $\mathbf{K}_{k}^{(i)}$} & Matrix add & $(M \times M)+(M \times M)$ & $O\left(N_{p} M^{2}\right)$ \\
\hline & Matrix inverse & $M \times M$ & $O\left(N_{p} M^{3}\right)$ \\
\hline & Matrix multiply & $(L \times M) \times(M \times M)$ & $O\left(N_{p} L M^{2}\right)$ \\
\hline Innovation $\mathbf{E}_{k, j}^{(i)}$ & Vector add & $(M \times 1)+(M \times 1)$ & $O\left(N_{p} N_{e} M\right)$ \\
\hline \multirow[t]{2}{*}{ Correction $\mathbf{x}_{k, j}^{(i), a}$} & Matrix multiply & $(L \times M) \times(M \times 1)$ & $O\left(N_{p} N_{e} L M\right)$ \\
\hline & Matrix add & $(L \times 1)+(L \times 1)$ & $O\left(N_{p} N_{e} L\right)$ \\
\hline Analysis ensemble mean $\overline{\mathbf{x}}_{k}^{(i), a}$ & Vector add & $(L \times 1)+(L \times 1)$ & $O\left(N_{p} N_{e} L\right)$ \\
\hline Analysis ensemble covariance matrix $\hat{\mathbf{P}}_{k}^{(i), a}$ & Vector multiply and add & $(L \times 1) \times(1 \times L)$ & $O\left(N_{p} N_{e} L^{2}\right)$ \\
\hline Particle update & Other & $(L \times 1)+(L \times 1)$ & $O\left(N_{p} L \mu\right)$ \\
\hline Weight calculate & Scalar multiply (M times) & $1 \times 1$ & $O\left(N_{p} M\right)$ \\
\hline State estimation & Vector add & $(L \times 1)+(L \times 1)$ & $O\left(N_{p} L\right)$ \\
\hline
\end{tabular}

\section{CONCLUSIONS}

In this paper, the time-evolving SSP was inverted sequentially by assimilating measurements of the complex acoustic field on a VLA using the PF, EnKF, and EnKPF.

Two examples with the range-independent environments were given, where the environments simulated from the PRIMER experiment and the ASIAEX ECS 2001 experiment were used to test the efficiency of the inversion schemes.

It is known that the PF can perform nonlinear and nonGaussian tracking, but it has the problem of sample degeneracy induced by the mismatch between the proposal distribution and the PPD. The EnKF can obtain the PPD of the state of the modeled nonlinear system according to Bayesian theory. The EnKPF employs the PPD of the EnKF as the proposal distribution of the $\mathrm{PF}$ to improve the performance of the PF. This makes the EnKPF an attractive complement to other techniques used in geoacoustic inversion, where the nonlinearity between the environmental parameters and the measured acoustic field is typically encountered. The disadvantage of the EnKPF is that it inevitably leads to a higher computational cost because of the combination of the EnKF and the PF. Simulation results showed that the EnKPF outperformed the PF and the EnKF over the whole tracking period of SSPs for different measured array SNRs. The comparison was also made for the case of a fast changing SSP, which confirms that the EnKPF is the best filter, as seen by evaluating the depth-integrated RMSE and the timeaveraged and depth-integrated RMSE. The simulations also showed that rapid variations can cause the filter to diverge.

It should be pointed out that although the EnKPF has the best accuracy in the tracking of the SSP, its computational complexity is greatly increased because of the combination of the PF and the EnKF. In practice, the best choice of the filter performance and the running time for a given hardware system is always a matter of trade-offs.

Additionally, the coherent processing of multiple frequency bins was also tested. It showed that the decrease of the performance of the EnKPF induced by the reduction of the number of hydrophones on the VLA could be compensated by employing coherent processing of multiple frequency bins, as proposed in Ref. 37. This constitutes an important guideline in practical implementation.

Finally, this simulation may be extended to track rangedependent sound speed fields and moving sources. It also could be a part of a larger tomography system. Considering multiple sensors with different types of ocean measurements, this method may be an interesting way to reconstruct a fourdimensional environment for underwater sensor networks. These topics should be further explored.

\section{ACKNOWLEDGMENTS}

The paper was much improved by the suggestions of the anonymous reviewers. The authors would like to express their great appreciation to Dr. James F. Lynch for many valuable suggestions to improve the content of this paper. They would also like to thank Dr. Wen Xu and Dr. Gopu R. Potty for the data of the PRIMER experiment, and Dr. Hangfang Zhao for the data of the 2001 ASIAEX. This work was supported by the National High Technology Research and Development Program of China (Grant No. 2012AA090901), the National Natural Science Foundation of China (Grant No. 61171147), and the State Key Laboratory of Acoustics, Chinese Academy of Sciences (Grant No. SKLOA201102).

${ }^{1}$ J. Zhou, X. Zhang, and P. H. Rogersand, "Resonant interaction of sound wave with internal solitons in the coastal zone," J. Acoust. Soc. Am. 90, 2042-2054 (1991). 
${ }^{2}$ J. R. Apel, M. Badiey, C. S. Chiu, S. Finette, R. Headrick, J. Kemp, J. F. Lynch, A. Newhall, M. H. Orr, B. H. Pasewark, D. Tielbuerger, A. Turgut, K. Von Der Heydt, and S. Wolf, "An overview of the 1995 SWARM shallow-water internal wave acoustic scattering experiment," IEEE J. Ocean. Eng. 22, 465-500 (1997).

${ }^{3}$ Y. T. Lin, T. F. Duda, and J. F. Lynch, "Acoustic mode radiation from the termination of a truncated nonlinear internal gravity wave duct in a shallow ocean area," J. Acoust. Soc. Am. 126, 1752-1765 (2009).

${ }^{4}$ J. Lynch, Y. T Lin, T. F. Duda, and A. E. Newhall, "Acoustic ducting, reflection, refraction, and dispersion by curved nonlinear internal waves in shallow water," IEEE J. Ocean Eng. 35, 12-27 (2010).

${ }^{5}$ T. F. Duda, J. M. Collis, Y.-T. Lin, A. E. Newhall, J. F. Lynch, and H. A. DeFerrari, "Horizontal coherence of low-frequency fixed-path sound in a continental shelf region with internal-wave activity," J. Acoust. Soc. Am. 131, 1782-1797 (2012).

${ }^{6}$ J. R. Daugherty and J. F. Lynch, "Surface wave, internal wave, and source motion effects on matched field processing in a shallow water waveguide," J. Acoust. Soc. Am. 87, 2503-2526 (1990).

${ }^{7}$ J. L. Krolik, "Matched-field minimum variance beamforming in a random ocean channel," J. Acoust. Soc. Am. 92, 1408-1419 (1992).

${ }^{8}$ D. R. Jackson and T. E. Ewart, "The effect of internal waves on matchedfield processing,” J. Acoust. Soc. Am. 96, 2945-2955 (1994).

${ }^{9}$ S. E. Dosso and M. J. Wilmut, "Data uncertainty estimation in matchedfield geoacoustic inversion,” IEEE J. Ocean. Eng. 31, 470-479 (2006).

${ }^{10}$ N. M. Patrikalakis, J. J. McCarthy, A. R. Robinson, H. Schmidt, C. Evangelinos, P. J. Haley, S. Lalis, P. F. J. Lermusiaux, R. Tian, W. G. Leslie, and $\mathrm{W}$. Cho, "Towards a dynamic data driven system for rapid adaptive interdisciplinary ocean forecasting," in Dynamic Data Driven Application Systems, edited by F. Darema (Kluwer Academic, Amsterdam, 2006), pp. $34-55$.

${ }^{11}$ W. Munk and C. Wunsch, "Ocean acoustic tomography: A scheme for large scale monitoring,” Deep-Sea Res., Part A 26, 123-161 (1979).

${ }^{12}$ W. Munk, P. Worcester, and C. Wunsch, Ocean Acoustic Tomography (Cambridge Univ. Press, Cambridge, UK), Chaps. 6-7, pp. 222-322 (1995).

${ }^{13} \mathrm{~J}$. P. Hermand and P. Gerstoft, "Inversion of broad-band multitone acoustic data from the Yellow Shark summer experiments," IEEE J. Ocean. Eng. 21, 324-346 (1996).

${ }^{14} \mathrm{P}$. Gerstoft and D. F. Gingras, "Parameter estimation using multifrequency range-dependent acoustic data in shallow water," J. Acoust. Soc. Am. 99, 2839-2850 (1996).

${ }^{15}$ S. E. Dosso, "Quantifying uncertainty in geoacoustic inversion. I. A fast Gibbs sampler approach,” J. Acoust. Soc. Am. 111, 129-142 (2002).

${ }^{16}$ S. E. Dosso and P. L. Nielsen, "Quantifying uncertainty in geoacoustic inversion. II. Ap-plication to broadband, shallow-water data," J. Acoust. Soc. Am. 111, 143-159 (2002).

${ }^{17}$ J. V. Candy and E. J. Sullivan, "Model-based environmental inversion: A shallow water ocean application," J. Acoust. Soc. Am. 98, 1446-1454 (1995).

${ }^{18}$ R. E. Kalman, "A new approach to linear filter and prediction problems," Trans. ASME J. Basic Eng. 82, 35-45 (1960).

${ }^{19} \mathrm{H}$. Cox, "On the estimation of state variables and parameters for noisy dynamic systems," IEEE Trans. Autom. Control AC-9, 5-12 (1964).

${ }^{20} \mathrm{G}$. Evensen, "The ensemble Kalman filter: Theoretical formulation and practical imple-mentation,” Ocean Dyn. 53, 343-367 (2003).

${ }^{21}$ P. L. Houtekamer and, H. L. Mitchell, "Ensemble Kalman filtering," Q. J. R. Meteorol. Soc. 131, 3269-3290 (2005).

${ }^{22}$ A. Doucet, S. Godsill, and C. Andrieu, "On sequential Monte Carlo sampling methods for Bayesian filtering,” Stat. Comput. 10, 197-208 (2000).
${ }^{23}$ M. Sanjeev, S. Maskell, N. Gordon, and T. Clapp, "A tutorial on particle filters for online nonlinear/non-Gaussian Bayesian tracking," IEEE. Trans Signal Process. 50, 174-188 (2002).

${ }^{24}$ C. Yardim, P. Gerstoft, and W. S. Hodgkiss, "Tracking of geoacoustic parameters using Kalman and particle filters," J. Acoust. Soc. Am. 125, 746760 (2009).

${ }^{25}$ O. Carrière, J. P. Hermand, J. C. Le Gac, and M. Rixen, "Full-field tomography and Kalman tracking of the range-dependent sound speed field in a coastal water environment," J. Mar. Syst. 78, 382-392 (2009).

${ }^{26} \mathrm{O}$. Carrière, J. P. Hermand, and J. V. Candy, "Inversion for time-evolving sound-speed field in a shallow ocean by ensemble Kalman filtering," IEEE. J. Ocean. Eng. 34, 586-602 (2009).

${ }^{27}$ C. Yardim, P. Gerstoft, and W. S. Hodgkiss, "Geoacoustic and source tracking using particle filtering: Experimental results," J. Acoust. Soc. Am. 128, 75-87 (2010).

${ }^{28}$ C. Yardim, Z.-H. Michalopoulou, and P. Gerstoft, "An overview of sequential Bayesian filtering in ocean acoustics," IEEE J. Ocean Eng. 36, 73-91 (2011).

${ }^{29}$ J. Mandel and J. D. Beezley, "An ensemble Kalman particle predictor corrector filter for non-Gaussian data assimilation," Lect. Notes Comput. Sci. $\mathbf{5 5 4 5}, 470-478$ (2009).

${ }^{30}$ G. van Delft, G. Y. El Serafy, and A. W. Heemink, "The ensemble particle filter (EnPF) in rainfall-runoff models," Stochastic Environ. Res. Risk Assess. 23, 1203-1211 (2009).

${ }^{31}$ R. Chen and J. S. Liu, "Mixture Kalman filters," J. R. Stat. Soc. Ser. B (Stat. Methodol.) 62, 493-508 (2000).

${ }^{32}$ L. R. LeBlanc and F. H. Middleton, "An underwater acoustic sound velocity data model," J. Acoust. Soc. Am. 67, 2055-2062 (1980).

${ }^{33}$ G. Potty, J. Miller, J. Lynch, and K. Smith, "Tomographic inversion for sediment parameters in shallow water," J. Acoust. Soc. Am. 108, 973-986 (2000).

${ }^{34} \mathrm{~W}$. Xu and H. Schmidt, "System-orthogonal functions for sound speed profile perturbation,” IEEE J. Oceanic Eng. 31, 156-169 (2006).

${ }^{35}$ P. H. Dahl, R. Zhang, J. Miller, L. Bartek, Z. Peng, S. Ramp, J.-X. Zhou, C.-S. Chiu, J. Lynch, J. Simmen, and R. C. Spindel, "Overview of results from the Asian Seas International Acoustics Experiment in the East China Sea," IEEE J. Oceanic Eng. 29, 920-928 (2004).

${ }^{36}$ M. B. Porter, "The KRAKEN normal mode program," Technical Report, SACLANT Undersea Research Center, La Spezia, Italy, 1991.

${ }^{37}$ J. P. Hermand and W. I. Roderick, "Acoustic model-based matched filter processing for fading time-dispersive ocean channels: Theory and experiments," IEEE. J. Ocean. Eng. 18, 447-465 (1993).

${ }^{38}$ C.-S. Chiu, F. L. Bahr, Y.-Q. Qi, P. H. Dahl, J. Miller, J. F. Lynch, R. Zhang, and J.-X. Zhou, "The shelf-edge frontal structure in the central East China Sea and its impact on low-frequency acoustic propagation," IEEE J. Oceanic Eng. 29, 1011-1031 (2004).

${ }^{39}$ J. Yang, S. Zhou, J.-X. Zhou, and J. F. Lynch, "Internal wave characteristics at the ASIAEX site in the East China Sea," IEEE. J. Ocean. Eng. 29, 1054-1060 (2004).

${ }^{40}$ Z. Peng, J.-X. Zhou, P. H. Dahl, and R. Zhang, "Seabed acoustic parameters from dispersion analysis and transmission loss in the East China Sea," IEEE J. Oceanic Eng. 29, 1038-1045 (2004).

${ }^{41}$ J. H. Miller, L. R. Bartek, G. R. Potty, D. Tang, J. Y. Na, and Y. Qi, "Sediments in the East China Sea," IEEE J. Oceanic Eng. 29, 940-951 (2004).

${ }^{42}$ R. Karlsson, T. Schon, and F. Gustafsson, "Complexity analysis of the marginalized particle filter," IEEE Trans. Signal Process. 53, 4408-4411 (2005). 